\title{
SCALA e Siesta Cloud: uma integração para aplicações homeschooling visando a inclusão
}

\author{
Liliana Maria Passerino¹, Maria Rosangela Bez ${ }^{1}$, João Carlos Gluz ${ }^{2}$, Enrique \\ Garcia $^{3}$, J. Miguel Ramirez ${ }^{3}$, Carlos de Castro ${ }^{3}$ \\ ${ }^{1}$ CINTED - UFRGS - Porto Alegre - RS - Brasil. \\ ${ }^{2}$ PIPCA -UNISINOS - São Leopoldo - RS - Brasil \\ ${ }^{3}$ CITEC - Universidad de Córdoba (UCO) - Córdoba - Espanha \\ liliana@cinted.ufrgs.br, bezrosangela@gmail.com, jcgluz@unisinos.br, \\ egsalcines@uco.es, josemiguel@cpmti.es, carlosdecastrolozano@gmail.com
}

\begin{abstract}
This paper describes the integration of two platforms for use with people in situations of dependency, one focused on the alternative communication destinated to individuals with communication deficits, with an emphasis on Autism Spectrum Disorder and the other for development of various activities with emphasis on homecare and homeschooling. Both platforms developed and research for more than five years, they integrate from a bi-national project between Spain and Brazil that describes the work as well as its first results.
\end{abstract}

Resumo. O presente artigo descreve a integração de duas plataformas para uso com pessoas em situação de dependência, uma voltada para a comunicação alternativa destinada a sujeitos com déficits de comunicação, com ênfase em Transtornos do Espectro Autista e a outra para desenvolvimento de atividades diversas, com ênfase em homecare e homeschooling. Ambas plataformas desenvolvidas e com pesquisas ha mais de 5 anos, integram-se a partir de um projeto binacional entre Espanha e Brasil que descreve-se no trabalho, assim como seus primeiros resultados.

\section{Introdução}

No processo de inclusão escolar, muitas vezes os alunos ficam impedidos de participar por longos períodos do convívio escolar, na maioria das vezes por questões de saúde decorrente de sua condição. Em outras circunstâncias, o processo de inclusão num turno escolar pode não ser suficiente para as necessidades especiais que esses alunos apresentam. Em ambas situações utilizar serviços digitais para um acompanhamento mais intensivo pode ser uma proposta de inclusão via homeschooling, principalmente para aqueles que apresentam sérias dificuldades na interação social (como no caso de sujeitos com Transtornos Globais do Desenvolvimento, entre os quais encontra-se a Síndrome de Autismo, assim como no caso de sujeitos com graves dificuldades motoras que envolvem o uso da fala). Habilidades de comunicação são fundamentais no desenvolvimento da interação social, pois possibilitam estabelecer reciprocidade e construir a intersubjetividade entre os participantes. Pessoas que apresentam déficits na comunicação precisam muitas vezes utilizar meios complementares, suplementares ou ampliadores de comunicação, denominados de Comunicação Alternativa (CA) de forma a garantir condições para o estabelecimento do processo de interação e comunicação. A CA é uma área interdisciplinar que objetiva desenvolver e estudar mecanismos, instrumentos e metodologias para complementar, suplementar ou aumentar o potencial de comunicação das pessoas. Nesse âmbito, o projeto SCALA já demonstrou o potencial para o desenvolvimento da comunicação em sujeitos com autismo (Passerino, 2013, Bez e Passerino, 2009). Agora, a proposta de integração com a plataforma Siesta visa eficiência em processos de homecare. Nosso intuito é discutir como essa integração pode potencializar processos de inclusão escolar de sujeitos com Transtorno do Espectro 
Autista complementados com homeschooling .

\section{SCALA}

O SCALA - Sistema de Comunicação Alternativa para Letramento de Pessoas com Autismo - iniciado em 2009, configurou-se como um plano articulado de trabalho em investigação, desenvolvimento tecnológico e formação que ao longo dos anos permitiu desenvolver uma solução tecnológica testada e utilizada em diferentes contextos. $\mathrm{O}$ SCALA objetiva apoiar o processo do desenvolvimento da linguagem em sujeitos com autismo e com déficits de comunicação, embasado epistemologicamente na teoria sócio-histórica. A versão inicial resultou num software que contava apenas com um módulo para produção de pranchas de comunicação acompanhado de uma proposta de uso com estratégias de interação e comunicação para crianças com autismo (Avila et al., 2011). A primeira versão do sistema foi testada num estudo de caso de um sujeito com autismo de 5 anos de idade em dois anos (Passerino et al., 2010; Avila et al., 2011) .

O SCALA 2.0 (http://scala.ufrgs.br/) inicia-se em $2011 \mathrm{com}$ a proposta de pensar numa solução multiplataforma do sistema especialmente para atender a demande de aplicativos para tablet. Todo desenvolvimento foi acompanhado e balizado com um estudo de caso (multicaso) com três crianças de 3 a 4 anos de idade com Autismo ao longo de dois anos. Esse estudo desenvolveu-se em 3 contextos diferentes: escola, familia e laboratório na universidade. O mesmo possui dois módulos: prancha (figura 1a) e histórias(figura 1b ) e foi desenvolvido sob as licenças GNU e Creative Commons para garantir seu conteúdo aberto. Os pictogramas utilizados no sistema foram, em sua maioria, desenvolvidos pelo grupo ARASAAC ${ }^{1}$. Com a utilização destas imagens e de imagens próprias, o SCALA conta com mais de 4000 (quatro mil) imagens, divididas nas categorias: Pessoas, Objetos, Natureza, Ações, Alimentos, Sentimentos, Qualidades e Minha Imagens, onde o usuário tem a opção de inserir imagens próprias no sistema. No módulo Prancha, há espaços estáticos na tela onde o usuário tem a possibilidade da escolha de um layout para construção de pranchas simples (figura 1a) até mais elaboradas, já no módulo história os espaços são livres para inserção de múltiplas imagens e elementos textuais (figura $1 \mathrm{~b}$ ). $\mathrm{O}$ módulo prancha consiste num editor de pranchas de comunicação e seu visualizador com sintetizador de voz embutido. As pranchas de comunicação são recursos de tecnologia assistiva que visam facilitar a comunicação entre pessoas com déficit na comunicação e outros participantes. As pranchas possuem layouts básicos escalonáveis que permitem que todo usuário crie suas próprias pranchas de forma simples e direta. Cada figura de uma prancha é editável, permitindo mudar sua legenda e som a partir de um menu sensível ao contexto. O módulo narrativas visuais serve para a construção de histórias, com condições flexíveis para elaboração das mesmas. Possui diversificados layouts que proporcionam um grau maior ou menor de complexidade. A tela possui um espaço em branco onde é possível além da inserção de imagens, editá-las. Estas podem ser sobrepostas, aumentadas ou diminuídas de tamanho, invertidas ou excluídas. Há a possibilidade de colocação de cor de fundo ou cenário. Também, há uma categoria de balões de conversação, sendo possível editá-los para inserção de pequenos diálogos. É possível escrever a história ou gravá-la, quando a história for reproduzida o sintetizador de voz irá ler o que foi digitado, caso contrário a gravação será reproduzida.

Durante todo o processo de desenvolvimento, diversas pesquisas foram desenvolvidas envolvendo crianças entre 3 e 5 anos com autismo, suas famílias e os professores das escolas onde as crianças encontravam-se incluídas o que nos permitiu um Design Centrado em Contexto de Uso (Passerino, 2013) para identificar pontos a serem melhorados e re-projetados. No ano de 2012, iniciou-se o desenvolvimento do modo de varredura a fim de contemplar sujeitos com deficiência motora. Esta nova funcionalidade surge como uma necessidade identificada nas pesquisas anteriores. Os sistemas de varredura existentes são todos pensados para público adulto, assim é importante projetar um sistema de varredura dirigido ao público infantil, definido como um sistema de

1 O banco de imagens utilizados no ambiente foram traduzidos dos símbolos pictográficos de CATEDU (http://catedu.es/arasaac/) sob a licença Creative Commons. 
varredura automática que pode ser ativado ou desativado facilmente e com configurações como tempo de intervalo, cor de destaque e som.

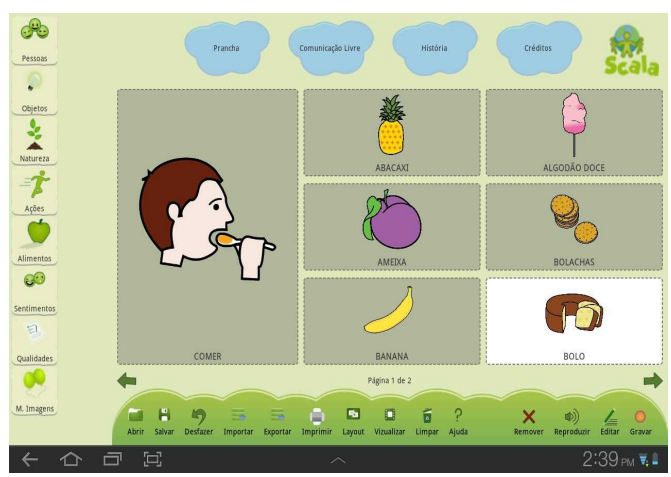

Figura 1 a - Módulo Prancha

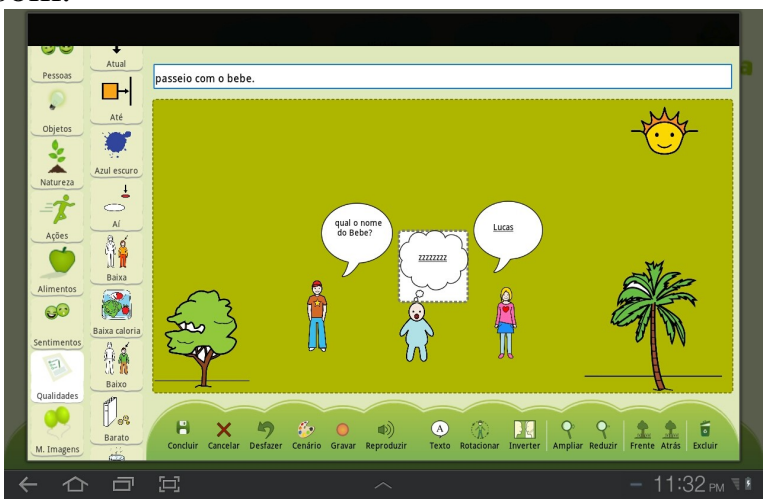

Figura 1 b Módulo História

Figura 1: Interface Scala

\subsection{SIESTA Cloud}

O Sistema Integrado de e-Serviços e Tecnologias de Ajuda na nuvem: SiestaCloud utiliza técnicas de Inteligencia Ambiental para possibilitar a integração em ambientes ubíquos como lares, hospitais, centros comunitários, escolas, etc.) onde os serviços de internet aliados a novos dispositivos de interface permitam um uso natural, integrado e não invasivo (XXX, 2011). SiestaCloud é o resultado de mais de 25 anos de investigação e desenvolvimento do grupo EATCO da Universidade de Córdoba e consiste numa plataforma na nuvem complementada com um sistema operativo Siesta local baseado em GNU/linux instalável na settop-box ou computador. Para De Castro (2011) SiestaCloud é um ecossistema, por se tratar de um conjunto de aplicações, sistema operacional e plataforma na nuvem cujo objetivo principal é dar condições de desenvolvimento a pessoas em situação de dependência como por exemplo idosos, pessoas com deficiência, pessoas hospitalizadas, entre outros. Por esse motivo, o SiestaCloud foi desenvolvido seguindo as normas internacionais para acessibilidade e com critérios de usabilidades rigorosamente testados (Bevan, 2012). Seus aplicativos potencializam a interação com o ambiente e permite desde o controle domótico até as interações mais tradicionais com internet. Por ser uma plataforma interoperável é possível utilizá-la a partir de qualquer dispositivo como computador, tablet, smartphone ou TV digital, independente de sistema operacional. Sua interação é embasada no uso de interfaces naturais como voz, gestos e movimentos, possibilitando desta forma acessibilidade total a partir do paradigma da inclusão. O SiestaCloud integra as últimas tendências tecnológicas de interação pessoa-computador, computação ubiqua, computação na nuvem, interação por gestos, voz e Internet das coisas, apresentando potencial para simplificar a enorme variedade de plataformas, ambientes, padrões e sistemas operacional existentes no mercado e facilitar a inclusão digital de um amplo público. A arquitetura base Siesta é formada por componentes de software e hardware, distribuídos em uma arquitetura cliente-servidor de três camadas (Figura 2): a camada de apresentação, com a interface de usuário tanto de entrada quanto de saída, a camada de lógica de negócio e a camada de acesso aos dados. A camada de apresentação suporta uma ampla gama de dispositivos de hardware incluindo, dentre outros: tablets, televisores digitais, PCs, settop-box IPTV, smartphones, servidores de streaming remoto para vídeos em tempo real e sob demanda, controles remoto, dispositivos de interação natural (SIN), dispositivos para chamadas de VoIP, módulos para controle domótico, dispositivos e sensores de telemedicina com conexão Bluetooth. A camada de negócio, provê a síntese de informação e é onde residem os programas que recebem, tratam e respondem as solicitações dos usuários. Esta camada é central na arquitetura Siesta se comunicando com a camada de apresentação, tanto com a interface de entrada quanto de saída, e com a camada de dados. A camada de dados é formada por um ou mais sistemas de gestão de bases de dados que realizam todo o armazenamento de 
dados, recebendo solicitações de armazenamento ou recuperação de informações da camada de negócios.

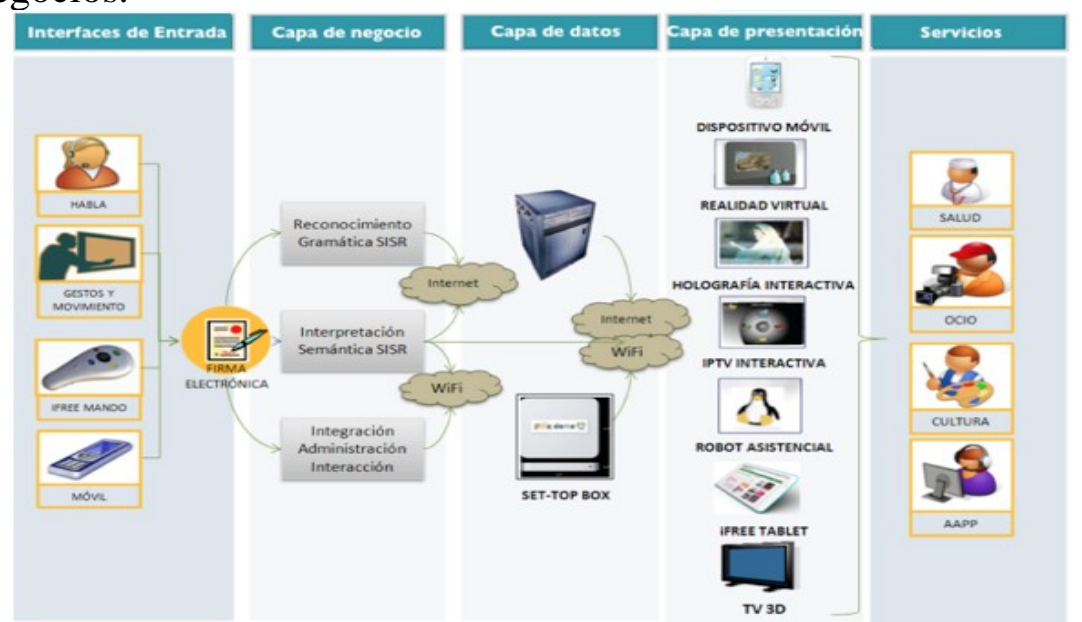

Figura 2: Arquitetura do Ecossistema Siesta

\section{TAC-ACCESS: integrando SCALA no Siesta}

O projeto TAC-ACCESS- Tecnologias de Apoio à Comunicação a partir de Interfaces acessíveis e multimodais para pessoas com deficiência e diversidade surge como um projeto de investigação e desenvolvimento tecnológico no âmbito das tecnologias de comunicação, tratamento da informação, aplicativos multimodais e tecnologias de interfaces aplicado ao campo da inclusão, pessoas em situação de dependência, no caso comunicacional, e aplica-se no campo da inclusão laboral e escolar. O projeto conta com o apoio da CAPES e do Ministério da Educação Espanhol, e permitiu desenvolver de forma integrada um modelo de cooperação internacional para desenvolvimento de tecnologias assistivas e de acessibilidade na perspectiva de Design Inclusivo a partir de atividades de pesquisa cooperativa e interdisciplinar. A principal linha investigativa é analisar como integrar um sistema de comunicação alternativa como o SCALA desenhado e projetado no Brasil num sistema multiplataforma como o Siesta: Como o Siesta pode ser usado em processos de inclusão escolar e laboral?; Que soluções e estratégias podem ser construídas a partir do uso do SCALA em processos de inclusão de pessoas com déficits de comunicação, especialmente na síndrome de autismo? Como usar realidade virtual e internet dos gestos para apoiar a inclusão de sujeitos com Transtorno do Espectro Autista? Que protocolos de análise de usabilidade podem ser construídos para atender a diversidade do Design Universal e a Tecnologia Assistiva?; Que soluções sócio-tecnológicas são perceptíveis a partir do uso do Siesta e SCALA em ambos países?; Que desenhos metodológicos podem ser elaborados para compreender as diferenças culturais no estudo das tecnologias de apoio a diversidade?; Como estabelecer processos de cooperação para elaboração de protocolos de usabilidade, acessibilidade, pesquisa etnográfica no desenvolvimento e aplicação de tecnologias de apoio que beneficiem países na América Latina e países de língua portuguesa, especialmente países africanos?,

No momento, o SCALA encontra-se integrado ao Siesta TV numa versão beta, sendo utilizado por ambos os grupos para validação de funcionalidades operacionais. Ajustes já foram feitos para garantir a integração no modelo de interface do Siesta porém mantendo as configurações essenciais. O sistema de leitor de tela embutido no SCALA, assim como o sistema de varredura foram retirados para incorporar as funcionalidades de acessibilidade de Siesta que aplicam-se a todos os aplicativos. A interface original do SCALA foi mantida, mas definiram-se cores para os ícones e menus do Siesta. A adaptação manteve-se fiel ao design universal, evitando "tecnologias especiais", pois nossa experiência mostra que quando desenhos se destinam a uma deficiência determinada, seu uso é mais limitado. Com isso, não queremos dizer que devemos ignorar as necessidades dos alunos com deficiência, mas sim que devemos pensar que ao focar o desenvolvimento no paradigma do déficit não estamos atendendo requisitos que garantam 
a inclusão. Assim, as tecnologias de apoio devem enfocar o Desenho Universal ou Para Todos (Mace et al., 2002), "levando em conta as necessidades e interesses de todos os possíveis usuários. Esta iniciativa enfatiza o indivíduo, facilita sua integração e busca a inclusão educativa e laboral." (Sanchez Montoya, 2006, p. nossa tradução) Esta perspectiva de desenvolvimento pensa o Desenho Universal para tecnologias de apoio a todas as pessoas, a despeito de suas deficiências.

Partindo de uma perspectiva metodológica sócio-histórica, nossa abordagem não contempla apenas o sujeito com deficiência, mas esse sujeito em interação em contextos sociais a partir de práticas culturais desenvolvidas pelos diferentes participantes, por meio de ações mediadoras. Essas são, portanto, nossas dimensões de análise e também as linhas de desenvolvimento do que denominamos de Design Centrado em Contextos de Uso (Bez, Passerino, 2011; Passerino, 2013) As diretrizes do DCC ajudam a planejar o desenvolvimento de Tecnologia Assistiva, sem focar apenas na dimensão funcional, mas incluir outras dimensões como a social e a cultural. Cada indivíduo "habita" diferentes contextos e é participante mais ou menos ativo de diferentes práticas culturais. Cada contexto configura relações e mediações possíveis que afetam de forma radical o uso pragmático que se fará da tecnologia como instrumento de mediação. O panorama traçado sobre os contextos constitui o nível macro de análise do fenômeno da comunicação dentro do espaço educativo ou laboral. Num nível micro as tríades sujeito-mediador, sujeito com déficits de comunicação e ações mediadoras são o ponto de partida para compreensão dos processos de mediação com tecnologias. Tal perspectiva metodológica colabora no desenvolvimento de recursos tecnológicos (entre eles o SCALA) de forma diferenciada que processos de desenvolvimento tradicionais, mesmo em aqueles processos embasados no Design Centrado no Usuário, no qual o usuário participa do processo de desenvolvimento. O SCALA foi desenvolvido na perspectiva de DCC, e sua integração com Siesta está sendo realizada da mesma forma: com pesquisas multicasos. No momento o primeiro caso está em andamento com mães de alunos que já utilizavam o SCALA com seus filhos, só que agora na plataforma Siesta. O segundo caso, iniciará em 2014 com aplicação deste produto em escolas espanholas e brasileiras em turmas inclusivas.

\section{Referencias}

Avila, B. G., Passerino, L.M., Reategui, E. B. SCALA um sistema de CAA focado no contexto do usuário. In: Anais do XXII SBIE, Aracaju/SE, 2011. p. 1663-1672.

Bevan, N.; Macleod, M. Usability measurement in context. Behaviour and Information Technology, n.13, v. $1 \& 2$.

Bez, M. R., Passerino, L. M. Applying Alternative and Augmentative Communication to an inclusive group. In: Anais do WCCE 2009, p. 164-174.

Bez, M. R. Passerino, L. M. Ações Mediadoras com uso de Comunicação Alternativa: Estudo de caso com Autismo. In: Anais do IV Congresso Brasileiro de Comunicação Alternativa, Rio de Janeiro, 2011, p. 1-5.

De Castro, C., Burón, F.J., Sainz, B., García, E. SIeSTA Project: Products and Results. LNCS. Springer, v. 6616, 2011.

Mace, R. et al. The Universal Design File: Designing for People of All Ages and Abilities, 2002.

Passerino, L. M.; Avila, B. G.; Bez, M. R. (2010). SCALA: um Sistema de Comunicação Alternativa para o Letramento de Pessoas com Autismo. RENOTE. Revista Novas Tecnologias na Educação, v. 1, p. 1-10, 2010.

Passerino, L. M. Comunicação alternativa, autismo e tecnologia: estudos de caso a partir do Scala. In: Miranda, T., Galvão Filho, T. (Org.). O professor e a educação inclusiva: formação, práticas e lugares. Salvador/BA: Editora UFB, 2012, v. 1, p. 217-240

Passerino, L.M. Anjos Tecnológicos na Torre de Babel: reflexões sobre o uso da Comunicação Alternativa em dispositivos móveis. In: Brito, M.C., Misquiatti, A.. (Org.). Transtornos do Espectro do Autismo e Fonoaudiologia: atualização multiprofissional em saúde e educação. Curitiba: CRV, 2013, v. 1, p. 209-228.

Sánches Montoya, R. Capacidades visibles, tecnologías invisibles: Perspectivas y estudio de casos. In: Rodríguez, J., Montoya, R., Soto, F. J. (coords.): Las tecnologías en la escuela inclusiva: nuevos escenarios, nuevas oportunidades. Murcia: Consejería de Educación y Cultura, 2006. 